\title{
Formation of a new dog population observed by pedigree and mtDNA analyses of the Polish Hovawart
}

\author{
Iwona Głażewska', Sylwia Zielińska² and Beata Prusak³ \\ 'Department of Plant Taxonomy and Nature Conservation, University of Gdańsk, Poland, 2Department of Genetics, \\ University of Gdańsk, Poland, ${ }^{3}$ Institute of Genetics and Animal Breeding PAS, Jastrzębiec, Poland
}

\begin{abstract}
The aim of the study was to evaluate changes in the gene pool of a dog population during the period of its formation. Pedigree and mtDNA analyses were performed on the Polish population of Hovawart dogs. A total of 192 litters of 93 dams and 115 sires were born between 1988 and 2008. Breeding began using Hovawarts imported mainly from the Czech Republic and Slovakia; however, the role of Western European dogs increased continually throughout the period analysed. No unfavourable effects caused by the limited size of the population were identified because of the constant inflow of new genes from abroad. The continual increase in the gene pool was indicated by all of the pedigree parameters analysed. Two different mtDNA haplotypes were found, and complete agreement between pedigree and molecular data was noted. The results of the analyses permit concluding that the process of formation of the new Hovawart population was also impacted by non-genetic factors that directly influenced the composition of gene pool.
\end{abstract}

Keywords: dog, Hovawart, pedigree analysis, mtDNA

\section{Introduction}

Many articles published in recent years have focused on pedigree analyses in dogs (Cole et al. 2004, Leroy et al. 2006, Calboli et al. 2008, Głażewska 2008, Ólafsdóttir \& Kristjánsson 2008, Leroy et al. 2009, Voges \& Distl 2009, Mäki 2010, Leroy \& Baumung 2011, and the review by Leroy 2011). The subjects of these studies were either rare local breeds classified as endangered or popular breeds bred in many countries. The databases used in these analyses differed in completeness and ranges of pedigree information. They comprised all information on a given breed (Głażewska 2008, Mäki 2010), or were composed using local sources of pedigree information, for example the database of the UK Kennel Club (Calboli et al. 2008) or the Société Centrale Canine (SCC) database (Leroy et al. 2006). As a rule, a group of dogs born within a defined period of a few years (a reference population) was the subject of analyses; however, some studies focused on populations observed for longer periods (Cole et al. 2004, Głażewska 2008, Voges \& Distl 2009, Mäki 2010).

The analyses have indicated a number of unfavourable occurrences in dog breeding, such as a high level of inbreeding, high disproportion in the breeding use of sires and strong imbalance in founder contributions to a gene pool, all of which might negatively influence the health condition of a given population (Cole et al. 2004, Leroy et al. 2006, Calboli et al. 
2008, Głażewska 2008, Ólafsdóttir \& Kristjánsson 2008, Oliehoek et al. 2009, Leroy et al. 2009, Voges \& Distl 2009, Mäki 2010). In the present study, we concentrated on changes in the gene pool of a pedigree dog population during the period of its formation. Our interest focused on genetic and non-genetic factors that influenced the decisions made by the breeders and whether the breeding policy was advantageous for the new population from a genetic point of view. Hovawart dogs, that have been bred in Poland since the 1980s, were used as a model population. The analyses were performed using pedigree data collected in the Polish archives, i.e. available for Polish breeders. The second goal of the study was to analyse mitochondrial DNA ( $m t D N A$ ) to evaluate mtDNA diversity over the span of the 21-year breeding period.

Hovawart dogs are a German working breed from the FCl (Fédération Cynologique Internationale) 2 group that originates from old guard dogs. Modern breeding began in 1924, and the breed was restored using Hovawart-type farm dogs and representatives of different breeds from $\mathrm{FCl} 1$ and 2 groups. The breed was recognised in 1936 under $\mathrm{FCl}$ number 190 . Breeding of this dog began in Poland with Britta von der Funkenmühle which was imported from East Germany (GDR), and her first litter by Ago vom Bretterkeller was born in 1988 at the Heland kennel.

\section{Material and methods}

Pedigree analysis of dogs, born in Poland between 1988 and 2008, was conducted using the pedigree database (further referred to as the Polish database) which comprised general information on the origin of the litters (parents, date of birth, kennel) and data on the origin of foreign parents from their four-generation pedigrees. The first part of the database, containing data for the 1988-2004 period, was published earlier by Głażewska (2006), and the remaining data were collected from the archive of the Polish Kennel Club (ZKwP). Not less than five generations of ancestors were known for each litter. The average pedigree length increased from 7.8 to 12.5 generations during the 21 years period studied, however, the average pedigree completeness increased only from 5.2 to 5.7 generations. This stemmed directly from the way the database was constructed i.e. using pedigree information exclusively from Polish archives.

Data on the number of puppies born between 2003 and 2008 and on dogs presented during 55 international and national Polish dog shows in 2009, which were published on two web sites (www.klub.hovawart.pl, www.zkwp.pl), were used in the study. Additionally, information from interviews with the Hovawart breeders and owners, and from the www. forum.hovawart.pl web site were used for the interpretation of the results, and these sources of information are quoted in the article as »breeder statements".

For the purpose of analysis, the 21-year period was divided into seven periods: 19881993, 1994-1998, 1999-2000, 2001-2002, etc. The basic breeding and genetic parameters were calculated. Ancestors with unknown parents in the Polish database were considered as founders. The founder contributions in a pedigree and the founder genome equivalent were computed using GENES v11.8 (Lacy 1998). The founder contribution is defined as the expected proportion of the population's gene pool that has descended from this founder (Lacy 1989), and it is equal to the value of the coefficient of relationship between the founder and its descendants. The founder genome equivalent (FGE) defined as the theoretical number of founders that would be required to provide the level of genetic diversity observed 
in the living population if the founders were all equally represented and had lost no alleles, was computed according to Ballou \& Lacy (1995). The total (FTOTAL) and 5-generation (F5) inbreeding coefficients (Wright, 1922), mean kinship (MK) (Malécot 1948), generation interval and the pedigree completeness level (which is given above) were computed using ENDOG v4.5 (Gutiérrez \& Goyache 2005).

The analysis of mtDNA diversity was performed using hair samples comprising 30-40 hairs each which were taken from the backs and tails of the dogs. In total, samples from 23 Hovawarts representing all dam lines and their branches were collected. Total genomic DNA from hair bulbs was extracted according to the standard organic procedure (Wilson et al. 1995). DNA amplification was performed using primers designed in this study with the Primer3 (Rozen \& Skaletsky 2000): CRDOGF (15372-15392): 5'GTA ACC GCC CTCCCT AAG AC3' and CRDOGR (1609616117): 5'TGT CCT GAA ACC ATT GAC TGA3. The PCR reaction was conducted in a GenAmp PCR System 9600 Thermal Cycler (Applied Biosystems, Foster City, CA, USA). The PCR products (660 base pair length) were purified using Microcon 100 microconcentrators (Amicon, USA) and sequenced with BigDye Terminator v1.1 Cycle Sequencing Kit (Amicon, Beverly, MA, USA) according to the user's manual. Purified sequencing products were separated in an Applied Biosystems 3130 DNA Analyser (Applied Biosystems, Foster City, CA, USA). The electrophoretic data were collected by the Data Collection v2.1. software and analysed by the Sequencing Analysis v3.0. software (Applied Biosystems, Foster City, CA, USA). The Hovawart sequences were compared to dog sequences deposited in GenBank. Phylogenetic analysis of the mtDNA haplotypes was performed using MEGA v4.0 software (Tamura et al. 2007).

\section{Results}

\section{Breeding data analysis}

Between 1988 and 2008 a total of 192 litters were born in Poland in 74 kennels, by 93 dams and 115 sires. Twenty-three dams and 91 sires originated from foreign breeding. Regarding the parents of Polish origin, they came from 68 litters and this number is equal to $50,7 \%$ of the total number of litters born until 2005. Differences in the breeding use of particular individuals were found. The majority of parents, $51.6 \%$ of the dams and $76.5 \%$ of the sires, had one litter only, and the maximum number of litters by one parent was 7 and 14, respectively. The length of the breeding use of females and males was similar. The average age of a dam and a sire at the birth of their first litter was 3.34 and 3.52 years, respectively, and the average generation interval between a dam and her litter was 4.47 years.

Significant differences in the number of litters born in particular kennels were observed. The majority of kennels (43) produced only one litter, and only 12 kennels gave five or more litters, with a maximum of 15 . In the kennels, which produced only one litter, $22.4 \%$ of the total number of litters was born. Only six bitches originating from these litters have been used in breeding, which is $8.6 \%$ of the total number of Polish breeding dams.

Incomplete data for the $2003-2008$ period which referred to $64 \%$ of the litters born, indicated that the average number of puppies in a single litter was 8.14 (from 2 to 13).

The analysis of parental origin indicated progressive changes in the population gene pool. During the initial years of breeding, with the exception of the first pair of parents that came from East Germany (GDR), breeding was dominated by individuals from the Czech Republic 
and Slovakia (CS) (Figure 1). Of the 23 imported bitches used in Polish breeding, 15 were from CS, three each from France and Germany, and one each from Hungary and Norway. The group of foreign sires was dominated by males from CS in the initial period, but in subsequent periods there was an increasing tendency to use males from Western European countries, mainly Germany. Breeders also decided to use dogs from other countries, such as France, Denmark, Finland and Norway, but this refers only to single individuals. The highest proportion of matings with Western European sires was noted in the 2003-2004 and 20052006 periods ( $45.5 \%$ and $45.3 \%$, resp.). The increasing tendency of foreign matings might be linked to the improvement of the economic situation in Poland (Figure 2).

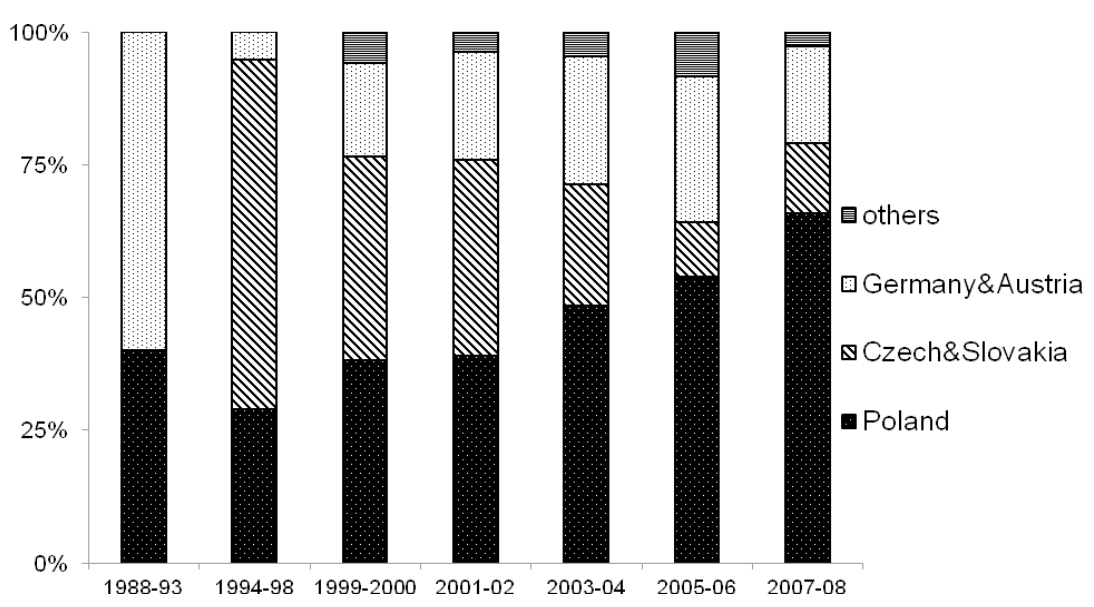

Figure 1

Parents of the litters according to the country of their origin

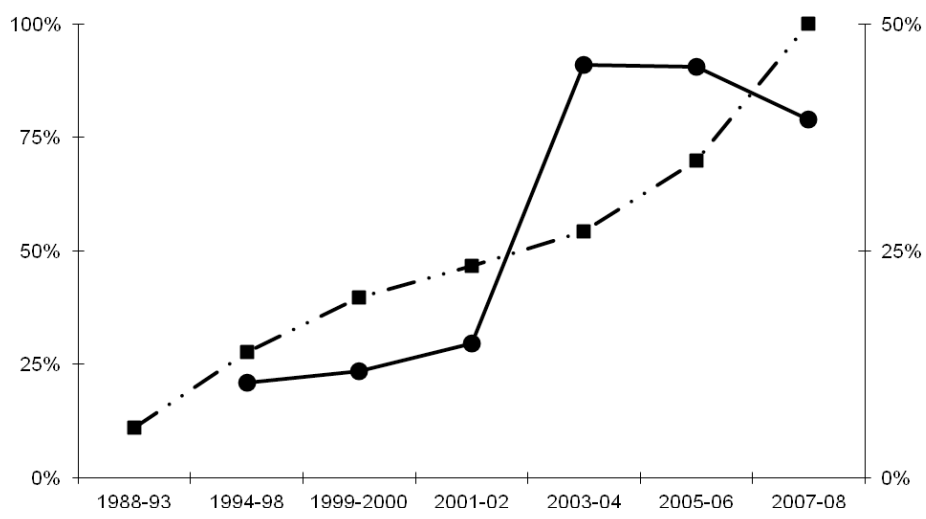

Figure 2

The percentage of Western European matings (solid line, right Y-axis), and the average salary in Poland, in USD, expressed as the proportion of the salary in 2008 (dashed line; left Y-axis), calculated on the basis of data published on the web sites of the National Bank of Poland and the Central Statistical Office. 
According to the Polish database, 23 imported breeding bitches represented 12 dam lines. The founders of these lines were nine bitches from the GDR, and single bitches from West Germany, Switzerland and Sweden. Figure 3 presents the dynamics of changes in the number of litters born in particular dam lines. The dominant line during the whole period was that of Adda vom Annatal which is represented mainly by descendants of Britta von der Funkenmühle, the first bitch used in Polish breeding.

The analysis of lists of dogs in Polish dog shows in 2009 indicated that only a limited number of dogs was shown (Figure 4). The highest proportion of dogs shown from a given litter was in the first or second year of life, i.e. they were shown mainly in junior classes. In comparison to the estimated total number of dogs born, this means that only about $24 \%$ of young dogs were judged in shows. A decreasing tendency in the number of dogs shown was linked to their age: older dogs were shown rarer.

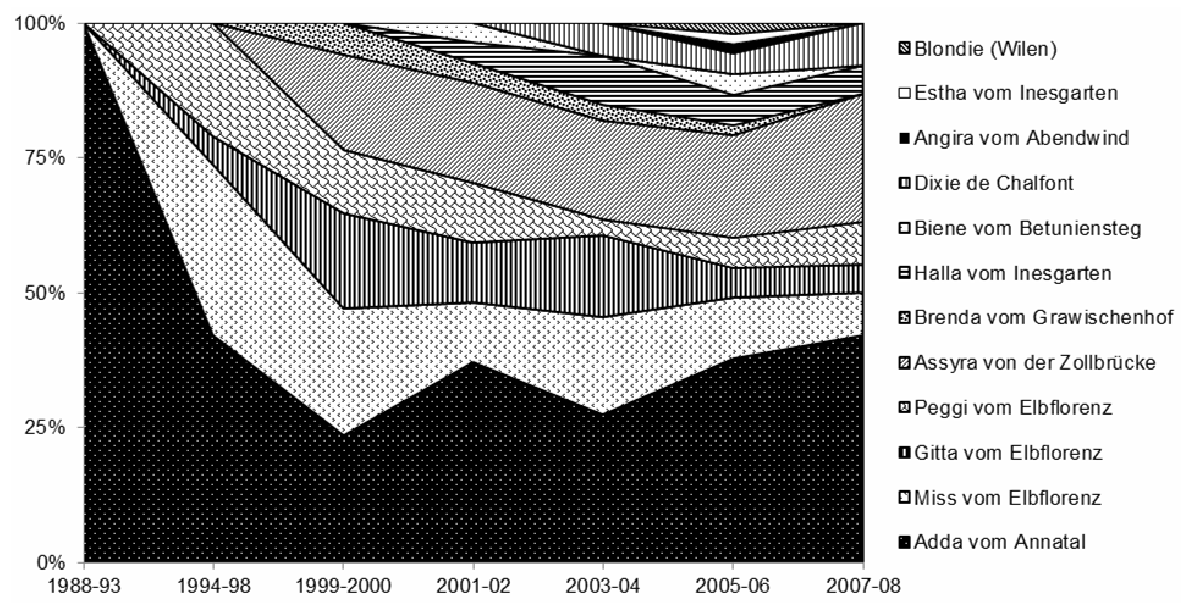

Figure 3

Proportion of the litters born in particular dam lines

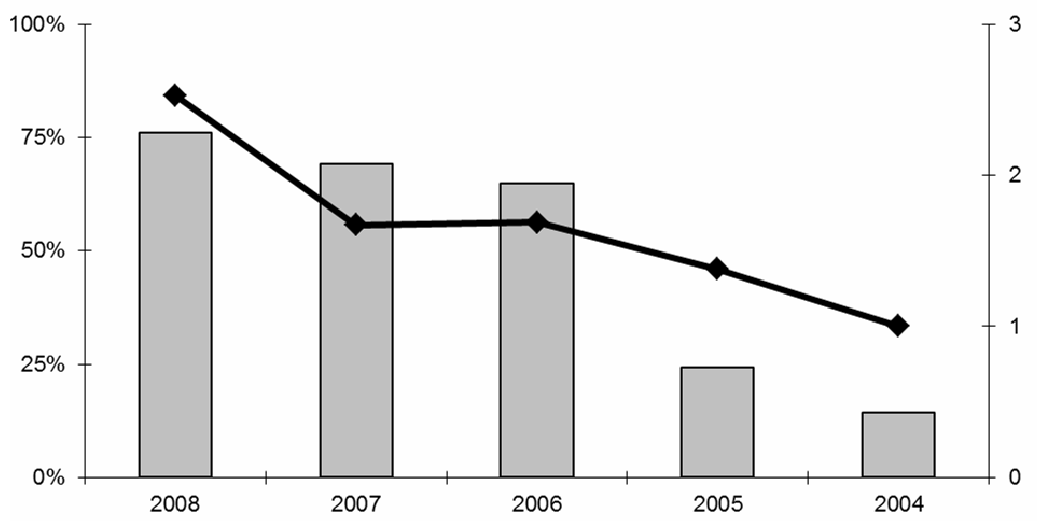

Figure 4

Dog shows in 2009: the percentage of litters with the representatives on Polish shows, according to a year of a litter birth (columns; left $Y$-axis), and the average number of dogs from one litter (line; right $Y$-axis) 


\section{Pedigree parameters analysis}

Table 1 presents detailed data of the pedigree analyses. A total of 203 founders (95 males and 108 females) were present in the pedigrees from the whole period analysed. The stable increasing tendency in founder numbers was accompanied by the loss of some founders from the pool and the appearance of new founders in the subsequent periods. Figure 5 presents the dynamics of change of founder contributions to the gene pool of the population. In the 19881993 period, seven founders each covered over $4.5 \%$ of the gene pool, the next nine founders covered from $2.5 \%$ to $4.5 \%$, and the contribution of the remaining 27 founders was lower than $2.5 \%$. In subsequent years, supplanting genes of the first founders by genes of new founders were noted. As the number of founders increased, the values of the inbreeding coefficient and mean kinship decreased (Table 1). The constant decreasing trend in the values of F5, which ranged from $6.05 \%$ in the first period to $0.60 \%$ in the last period, is worth particular notice.

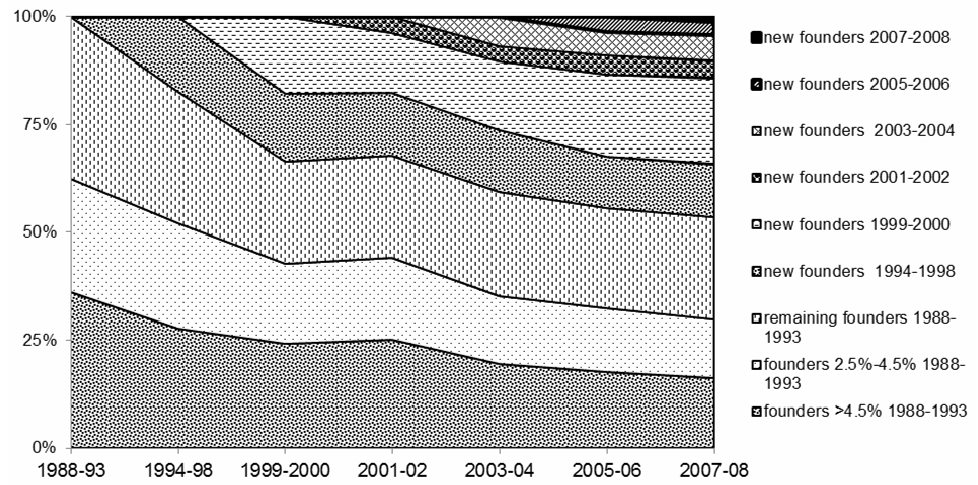

Figure 5

Founder contributions to the gene pool in the 1988-2008 period

Table 1

Basic parameters of pedigree analysis in Hovawart dogs bred in Poland

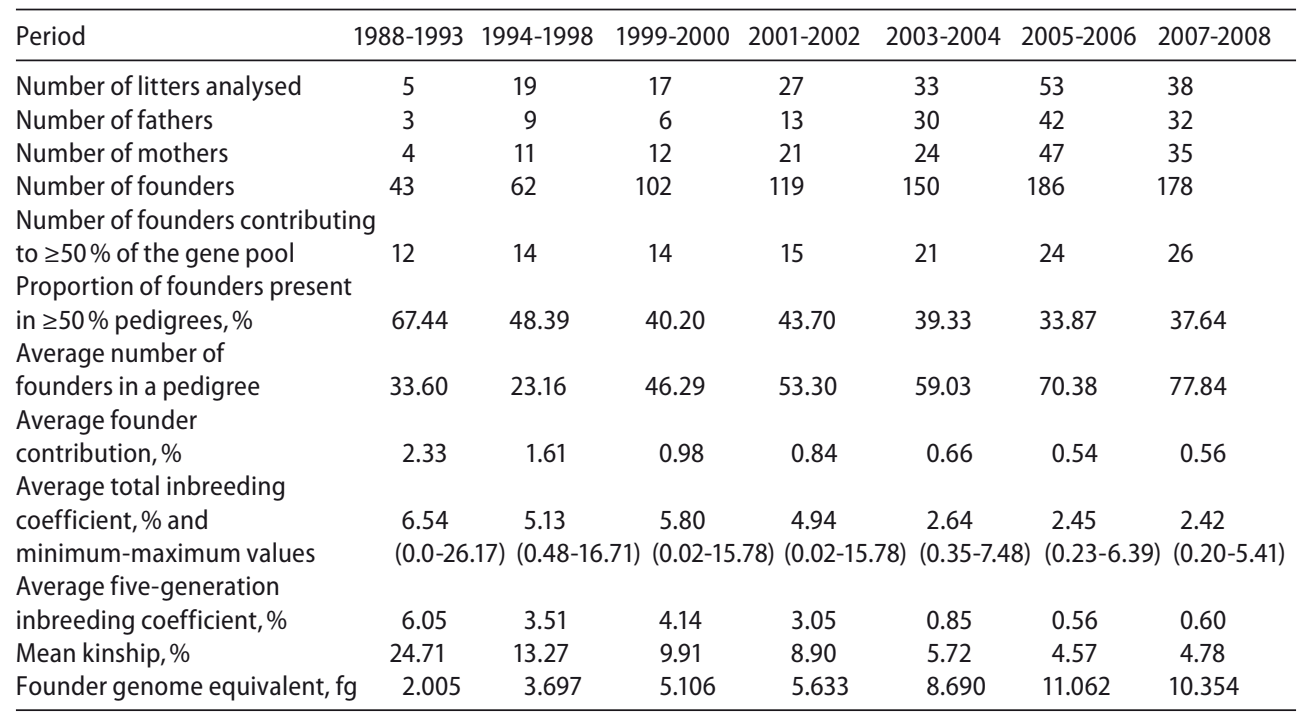




\section{Analysis of $m t D N A$}

Samples from representatives of all 12 dam lines and their main branches were studied. Two haplotypes, Ho1 and Ho2 (HM007196-HM007197 GenBank accession numbers), belonging to two clearly distinct haplogroups that correspond to clad A (Ho1) and clad B (Ho2) as determined by Savolainen et al. (2002), were noted. The Ho1 sequence differed from the reference sequence (U96639; Kim et al. 1998) by 15 nucleotides (14 transitions, one transversion), and the $\mathrm{Ho} 2$ sequence by six nucleotides (five transitions, one transversion). The sequences differed among themselves by 18 nucleotides. The Ho1 halpotype was found in the dam line that originated from Adda von Annatal and which was continued by two imported bitches (Britta von der Funkenmühle, Andromeda Queen Elsa). The Ho2 haplotype was found in the remaining 11 Polish lines.

Comparing the Hovawart sequences with those of other dog breeds we found some identical sequences (Table 2). The Ho1 haplotype is identical to the set of sequences of breeds representing different $\mathrm{FCl}$ groups. Among them is the St. Bernard, a breed recognised as one of the Hovawart founder breeds. The identical sequence was also found in the Golden Retriever, which exhibit significant phenotype similarity to blond-haired Hovawarts, even is not mentioned as a founder breed for Hovawarts. Only one identical sequence to the Ho2 haplotype was found in the Labrador Retriever breed till now.

Table 2

Haplotypes of Polish Hovawart dogs and identical 660 bp-long sequences found in other breeds.

\begin{tabular}{cccc}
\hline Hovawart haplotype & $\begin{array}{c}\text { GenBank acc. no. } \\
\text { of identical sequences }\end{array}$ & Breed & FCI group \\
\hline Ho1 & EU408260 & Welsh Corgi Cardigan & I \\
& DQ480500 & Shetland Sheepdog & I \\
EU408278 & Great Pyrenees & II \\
AY656743 & Saint Bernard & II \\
& EU223391* & Airedale Terrier & III \\
& EU408247 & Australian Terrier & III \\
& EU223558* & Fox Terrier & III \\
& AY656740 & Kerry Blue Terrier & III \\
& EU223711* & Rat Terrier & III \\
& EU408254 & Basset Hound & VI \\
& EU223565* & Golden Retriever & VIII \\
& EU408252 & Bolognese & IX \\
& EU408292 & Poodle & IX \\
& EU408301 & Tibetan Spaniel & IX \\
Ho2 & HM561534 & Labrador Retriever & VIII \\
\hline
\end{tabular}

*The shorter 628 bp region, 15458-16085 of the reference sequence, was concerned.

\section{Discussion}

The Polish population of Hovawart dogs is an interesting example of a new dog population. The population exhibited both similarities and differences in breeding parameters in comparison to breeds studied previously. Similarly to other breeds, occasional breeding prevailed and $58.1 \%$ of kennels had just one litter. One-litter kennels are typical of dog 
breeding, and occasional breeders were also noted frequently by Leroy et al. $(2007,2009)$, Calboli et al. (2008), Głażewska (2008) and Mäki (2010).

The generation interval of Hovawart (4.47) was relatively long in comparison with other breeds. For example, in Leroy et al. (2009) the generation interval in just 20 of 61 breeds representing different $\mathrm{FCl}$ groups was 4.5 or more. Next, the average generation interval was 3.8 in the breeds from the $\mathrm{FCl} 2$ group, which is of particular note since the Hovawart breed also belongs to this group. Leroy et al. explained this result by the shorter lifespan of the dogs and the early end of their reproductive capacity. Meanwhile, the prevailing opinion among Polish breeders is that breeding Hovawarts early is not recommended because the breed matures late. The result of this opinion is a late moment of the breeding entry both of Polish Hovawart males and females.

Significant differences were noted regarding the ratio of the number of dams to sires. The number of dams is generally higher than that of sires in dog breeding, and this is mainly because of the "popular sire effect» (Ostrander \& Kruglyak 2000), which refers to the high number of offspring that are fathered by the most desirable sires. Meanwhile, for Hovawarts the number of the dams was lower than the number of sires. This is the result of the high proportion of foreign matings and the almost unlimited choice of sires available to Polish breeders.

Over the span of 21 years of breeding, the gene pool increased continuously as indicated by all the pedigree parameters. Significant differences between $\mathrm{F}_{5}$ and $\mathrm{F}_{\text {TOTAL }}$ observed in recent years and the high proportion of litters with $\mathrm{F}_{5}=0 \%$, which resulted from intentional breeder decisions to avoid mating between relatives, are both noteworthy. This approach is not typical of dog breeding. According to Leroy et al. (2007), $24 \%$ of French breeders declared using close-breeding, and a similar tendency can be probably found in the breeding of the majority of dog breeds.

The constant inflow of new genes resulted in changes in the structure of the gene pool seen by the founder contributions. With a passage of time the contributions of the first population founders decreased as did disproportions in the contributions of particular founders. The trend is advantageous because high disproportion in the founder contributions to a gene pool is one of the most important issues in breeding such small populations of dogs (Leroy 2011). The increasing genetic diversity of the population was mainly the result of foreign matings. The use of newly imported bitches for breeding was significantly genetically less effective. The dam line established by the first imported bitch held the dominant position in relation to the number of litters born throughout the period analysed.

The high level of genetic diversity of the population was not reflected by mtDNA analysis and only two mtDNA sequences were found in the population. However, this number should be recognised as the typical number of haplotypes present in a single dog breed (Angleby \& Savolainen 2005, Pires et al. 2006). The number of haplotypes is not directly related to the size of the dog population but rather stems from breed history or breeding policy, e.g., whether the breeders ensure the continuation of particular dam lines or not.

Using additional pedigree information from the database http://www.working-dog.eu, we ascertained that the founders of 12 Polish lines originated from two founders of the breed, Dina (Geisler) (Adda von Annatal line) and Dina (Bruser) (remaining 11 lines), which were described as Hovawart farm dogs. Dina (Bruser) was born in 1923, and the date of birth of Dina (Geisler) is not given in this database but her daughter, Hova, was born in 1925. The results of the mtDNA analysis confirm that the pedigree data in the dam lines from almost 90 years of breeding are reliable. This is an important observation since similar analyses in horses have always led to 
deny some pedigree records (Hill et al. 2002, Kavar et al. 2002, Głażewska et al. 2007).

The interesting result of the analysis is the numerical supremacy of the Ho2 haplotype in the imported bitches. The question remains open of whether this haplotype also dominates in the world population of Hovawarts, or if its high frequency in the group of imported bitches is a founder effect stemming from breeder decision regarding imports.

One negative aspect noted in the breeding policy was the high imbalance in the chances of particular dogs transferring their genes to subsequent generations. Firstly, the chance of doing so depended on show participation. Only a small number of dogs were shown in dog shows and the remaining dogs are, in fact, a priori eliminated from the breeding. This follows from the Polish kennel regulations (http://www.zkwp.pl), according to which positive evaluations earned from adult show classes are required to receive breeding qualifications. Therefore, the decision of owners to show a given dog or not is crucially important for its breeding career. Show titles are also very important, and dogs with champion titles dominated breeding. According to the Polish database, of 22 Polish fathers of litters born since 1995, 19 were Polish champions. The significance of champion titles is economic since parents that are successful in shows determine the commercial success of Polish kennels in the market because buyers prefer puppies of champions (breeder statements).

In the population studied, the chances of bitches transferring their genes to subsequent generations also depended on their birthplaces. Bitches born in one-litter kennels were bred relatively more rarely. Since such litters originated from new and often genetically highly valuable matings, this is an especially unfavourable aspect of breeding. Moreover, this situation is difficult to explain in genetic terms because the parents of litters from onelitter kennels appeared to be of the same high quality, regarding to show titles and health condition, as were the parents from multi-litter kennels. This suggests that the reasons for this must lie with the subjective decisions of dog buyers. Our observations of the Hovawart breeding community indicate that persons with a greater knowledge of the breed and with precise plans as to dog shows, training and future breeding look for puppies at established kennels. Meanwhile, persons with less knowledge of the breed or those interested in acquiring a family dog buy puppies from kennels located closer to their homes or from those that are selling at lower prices (breeder statements). This stratification in buyer intention negatively impacted the dynamics of change in the gene pool of the population. Despite the high proportion of foreign matings and the use of many new dams in recent years, the enrichment and rebuilding of the gene pool has not progressed as expected based on the demographic data of the Polish population. This is clearly visible in Figure 3, which presents the dominant position of the first Polish dam lines in light of the number of litters born.

The composition of the gene pool of Polish Hovawarts was also influenced by economic factors. Initially, breeding animals were purchased in the Czech Republic and Slovakia where prices for puppies were lower than in Western Europe. As the economic situation in Poland improved, breeders began to use breeding material from Western Europe more frequently. The country of origin of breeding dogs plays an important role in the breeding of working dogs, such as Hovawarts, because in some countries breeding focuses on phenotypes rather than the behaviour traits of the dogs. This can be seen, for instance, in the different qualification criteria for breeding dogs in the German Rassezuchtverein für Hovawarthunde (RZV) (one dog show note and two high quality club performance tests, which were described in details 
by Boenigk et al. 2006) (http://www.hovawart.org) and in the Polish Kennel Club (three dog show notes and a standard performance test) (http://www.zkwp.pl). The superiority of show criterion over utility value in Polish breeding has contributed to the prevailing opinion that while Polish Hovawarts are handsome, they have weaker characters and are not as good workers as are German dogs (breeder statements).

Based on the present analysis and many years of observing Polish Hovawart breeding, we concluded that the breeding is significantly conditioned by non-breeding, economic and psychological factors. These begin with decisions to purchase eight-week-old puppies and end with the decision to begin and continue breeding. The personal preferences of breeders regarding phenotype and behavioural characteristics of the dogs, not always corresponding with the standards of the breed, are also very important. Equally important factors in dog breeding also include personal ambitions and relations among breeders. Unfortunately, friendships or animosities behind particular breeding decisions are immeasurable with genetic methods and so important aspect of dog breeding remains terra incognita.

\section{Acknowledgements}

We are grateful to the owners of Hovawart dogs for their help in collecting hair samples. This study was supported by the University of Gdańsk (BW/L 155-5-0345-9) and the Institute of Genetics and Animal Breeding in Jastrzębiec.

\section{References}

Angleby H, Savolainen P (2005) Forensic informativity of domestic dog mtDNA control region sequences. Forensic Sci Int 154, 99-110

Ballou JD, Lacy RC (1995) Identifying Genetically Important Individuals for Management of Genetic Variation in Pedigreed Populations. In: Ballou JD, Gilpin ME, Foose TJ (eds.) Population Management for Survival and Recovery. Analytical Methods and Strategies in Small Population Conservation, Columbia University Press, New York, NY, USA, 76-111

Boenigk K, Hamann H, Distl O (2006) [Analysis of environmental and genetic influences on the outcome of the juvenile and breeding performance tests for behaviour traits in Hovawart dogs]. Berl Münch Tierärztl Wochenschr 119, 258-269 [in German]

Calboli FCF, Sampson J, Fretwell N, Balding DJ (2008) Population structure and inbreeding from pedigree analysis of purebred dogs. Genetics 179, 593-601

Cole JB, Franke DE, Leighton EA (2004) Population structure of a colony of dog guides. J Anim Sci 82, 2906-2912

Głażewska I (2006) [The Stud Book of Hovawarts. Pedigree database 1988-2004]. Ed. Hovawart Club in Poland, Lamar Gdańsk, Poland [in Polish]

Głażewska I (2008) Genetic diversity in Polish hounds estimated by pedigree analysis. Livest Sci 113, 296-301

Głażewska I, Wysocka A, Gralak B, Prus R, Sell J (2007) A new view on dam lines in Polish Arabian horses based on mtDNA analysis. Genet Sel Evol 39, 609-619

Gutiérrez JP, Goyache F (2005) A note on ENDOG: a computer program for analysing pedigree information. J Anim Breed Genet 122, 172-176

Hill EW, Bradley DG, Al-Barody M, Ertugrul O, Splan RK, Zakahrov I, Cunningham EP (2002) History and integrity of thoroughbred dam lines revealed in equine mtDNA variation. Anim Genet 33, 287-294

Kavar T, Brem G, Habe F, Sölkner J, Dovč P (2002) History of Lipizzan horse maternal lines as revealed by mtDNA analysis. Genet Sel Evol 34, 635-648 
Kim KS, Lee SE, Jeong HW, Ha JH (1998) The complete nucleotide sequence of domestic dog (Canis familiaris) mitochondrial genome. Mol Phylogenet Evol 10, 210-220

Lacy RC (1989) Analysis of founder representation in pedigrees: founder equivalents and founder genome equivalents. Zoo Biol 8, 111-123

Lacy RC (1998) GENES. Version 11.8. Software Package for Genetic Analysis of Studbook Data

Leroy $G$ (2011) Genetic diversity, inbreeding and breeding practices in dogs: results from pedigree analyses. Vet J 189, 177-182

Leroy G, Rognon X, Varlet A, Joffrin C, Verrier E (2006) Genetic variability in French dog breeds assessed by pedigree data. J Anim Breed Genet 123, 1-9

Leroy G, Verrier E, Wisner-Bourgeois C, Rognon X (2007) Breeding goals and breeding practices of French dog breeders: results from the large survey. Rev Med Vet Toulouse 158, 496-503

Leroy G, Verrier E, Meriaux JC, Rognon X (2009) Genetic diversity of dog breeds: within-breed diversity comparing genealogical and molecular data. Anim Genet 40, 323-332

Leroy G, Baumung R (2011) Mating practices and the dissemination of genetic disorders in domestic animals, based on the example of dog breeding. Anim Genet 42, 66-74

Mäki K (2010) Population structure and genetic diversity of worldwide Nova Scotia Tolling Retriever and Lancashire Heeler dog populations. J Anim Breed Genet 127, 318-326

Malécot G (1948) [The Mathematics of Heredity]. Masson et Cie press, Paris, France [in French]

Ólafsdóttir GÁ, Kristjánsson T (2008) Correlated pedigree and molecular estimates of inbreeding and their ability to detect inbreeding depression in the Icelandic sheepdog, a recently bottlenecked population of domestic dogs. Conserv Genet 9, 1639-1641

Oliehoek PA, Bijma P, van der Meijden A (2009) History and structure of the closed pedigreed population of Icelandic Sheepdogs. Genet Sel Evol 41, 39

Ostrander EA, Kruglyak L (2000) Unleashing the canine genome. Genome Res 10, 1271-1274

Pires A, Ouragh L, Kalboussi M, Matos J, Petrucci-Fonseca F, Bruford MW (2006) Mitochondrial DNA sequence variation in Portuguese native dog breeds: diversity and phylogenetic affinities. J Hered 97, 318-330

Rozen S, Skaletsky JH (2000) Primer3 on the WWW for general users and for biologist programmers. In: Krawetz SA, Misener S (eds.) Bioinformatics Methods and Protocols: Methods in Molecular Biology. Humana Press, Totowa, NJ, USA, 365-386

Savolainen P, Zhang YP, Luo J, Lundberg J, Leitner T (2002) Genetic evidence for an East Asian origin of domestic dogs. Science 298, 1610-1613

Tamura K, Dudely J, Nei M, Kumar S (2007) MEGA4: Molecular Evolutionary Genetics Analysis (MEGA) software version 4.0. Mol Biol Evol 24, 1596-1599

Voges S, Distl $O$ (2009) Inbreeding trends and pedigree analysis of Bavarian mountain hounds, Hanoverian hounds and Tyrolean hounds. J Anim Breed Genet 126, 357-365

Wilson MR, Polanskey D, Butler J, DiZinno JA, Repogle J, Budowle B (1995) Extraction, PCR amplification, and sequencing of mitochondrial DNA from human hair shafts. Biotechniques 18, 662-669

Wright S (1922) Coefficients of inbreeding and relationship. Am Nat 56, 330-338

Received 22 June 2011, accepted 26 April 2012

Corresponding author:

Iwona Głażewska

email: i.glazewska@ug.edu.pl

Department of Plant Taxonomy and Nature Conservation, University of Gdańsk, Al. Piłsudskiego 46, 81-378 Gdynia, Poland 